\title{
Pour une réorientation fondamentale des politiques culturelles
}

The Necessity of setting up again cultural Policies

\section{Anne-Marie Autissier}

\section{(2) OpenEdition}

\section{Journals}

Édition électronique

URL : http://journals.openedition.org/questionsdecommunication/1784

DOI : 10.4000 /questionsdecommunication. 1784

ISSN : 2259-8901

Éditeur

Presses universitaires de Lorraine

\section{Édition imprimée}

Date de publication : 1 juillet 2008

Pagination : 239-250

ISBN : 978-2-86480-952-4

ISSN : 1633-5961

Référence électronique

Anne-Marie Autissier, "Pour une réorientation fondamentale des politiques culturelles », Questions de communication [En ligne], 13 | 2008, mis en ligne le 01 juillet 2010, consulté le 08 avril 2021. URL :

http://journals.openedition.org/questionsdecommunication/1784; DOI : https://doi.org/10.4000/ questionsdecommunication. 1784 


\title{
$>$ ÉCHANGES
}

\author{
ANNE-MARIE AUTISSIER \\ Centre d'études des mutations en Europe \\ Université Paris 8 Vincennes-Saint-Denis \\ amautissier@wanadoo.fr
}

\section{POUR UNE RÉORIENTATION FONDAMENTALE DES POLITIQUES CULTURELLES}

\begin{abstract}
Résumé. — Quoique les États membres de l'Union européenne soient des protagonistes ambigus des avancées en matière culturelle et artistique, il convient de reconnaitre les progrès accomplis depuis 1984, en matière de soutien au patrimoine, aux bibliothèques, au cinéma, à l'audiovisuel et aux arts du spectacle. II est aussi intéressant d'insister sur les concertations nombreuses qu'ont connues les États membres à la faveur de ces avancées institutionnelles. L'obtention de l'exception culturelle auprès de l'Organisation mondiale du commerce n'est pas le moindre résultat de ces concertations, même si son statut reste fragile et son application beaucoup trop partielle. Quant à la diversité culturelle, elle est reconnue comme partie intégrante des États de l'Union eux-mêmes, à la faveur des émergences régionales ouest-européennes mais aussi de l'élargissement. En 2007, la Commission européenne a proposé un Agenda culturel européen à l'ère de la mondialisation, incitant les États membres à se saisir de responsabilités en matière de coopération culturelle communautaire et internationale, via la Méthode ouverte de coordination (MOC) en y associant étroitement les échelons sub-nationaux. De plus, la Commission européenne souligne la nécessité d'un dialogue plus suivi avec la «société civile culturelle européenne » qui regroupe à ce jour plus de cent associations, fédérations et réseaux, fort actifs depuis les années 80 . Au-delà des programmes communautaires existants de coopération extérieure, l'articulation recommandée par la Commission entre coopération culturelle interne et extérieure de l'Union européenne, a ouvert la voie à des collaborations multilatérales susceptibles d'associer plus étroitement d'autres unions régionales à l'œuvre dans le monde : ANASE, MERCOSUR, etc. II reste que les conséquences anthropologiques de la numérisation restent sous-estimées par l'Union européenne. Loin de constituer un simple cadre d'adaptation à la conquête de nouveaux marchés, elle appelle de nouvelles formes de formation, de socialisation et de rémunération du travail artistique et intellectuel.
\end{abstract}

Mots clés. - Union européenne, États membres, Commission européenne, patrimoine, bibliothèques, cinéma, audiovisuel, arts du spectacle, exception culturelle, diversité culturelle, Organisation mondiale du commerce, régions d'Europe, élargissement, Méthode ouverte de coordination, société civile européenne, réseaux culturels, associations culturelles, fédérations culturelles, numérisation, ANASE, MERCOSUR, formation, propriété intellectuelle. 
L

es contributions de Jean Tardif et de Joëlle Farchy ici même jettent les fondements d'une nouvelle prise en compte des identités et échanges culturels contemporains dans le cadre de la mondialisation. Tous deux s'accordent à regretter le « nationalisme méthodologique » (Beck, 2004) des politiques culturelles - notamment en Europe--ainsi que l'inadéquation des modes de régulation proposés, liés à des approches sectorielles que les activités numériques mettent à mal.

Pour Jean Tardif, la dimension culturelle de la mondialisation crée un « nouvel écosystème » symbolique dans lequel les oligopoles du divertissement (américaines, mais aussi brésiliennes, indiennes, etc.) « offrent» au monde entier une « hyperculture globalisante », remettant en cause à la fois les possibilités locales d'expression identitaire et une certaine forme de « sécurité culturelle ». Selon Jean Tardif, l'adoption de la Convention UNESCO « sur la protection et la promotion de la diversité des expressions culturelles » apparaît comme une riposte trop faible au dispositif mis en place par l'Organisation mondiale du commerce (OMC). Par ailleurs, comme le fait justement remarquer Joëlle Farchy, les États membres de I'Union européenne se sont prononcés en faveur de l'exception culturelle dans le cadre des Accords de l'AGcs (en toutes lettres), principalement au bénéfice du cinéma et de l'audiovisuel, tout en faisant des concessions sur d'autres domaines, en particulier l'architecture. Force est de constater que l'exception culturelle est partielle, qu'elle peut être remise en discussion à la demande de l'un des États membres de l'OMc et que la Convention envisage un organe de règlement des conflits, somme toute facultatif. Pourtant, que de réunions, de négociations, de travail pour obtenir l'exception culturelle auprès de l'OMC, tout comme l'adoption de la Convention de l'UNESCO ! Sans doute doit-on se féliciter que les échanges culturels - même de façon partielle - aient donné lieu à des débats nourris et suivis par des citoyens de tous pays. Dans la phase actuelle, il convient donc d'insister sur le rôle politique - même modeste - de ces avancées. Dans le cadre d'un ouvrage dirigé par François Roche (2002), La culture dans les relations internationales, j'ai qualifié les États membres de l'Union européenne de «protagonistes ambigus » de la coopération culturelle européenne (Autissier, 2002). Certes, le rôle des politiques culturelles est particulièrement dépendant d'une certaine idée de l'État-nation et le paradigme un pays/une langue/un patrimoine et un répertoire national reste dominant et a, si j'ose dire, repris de la vigueur avec l'adhésion à l'Union européenne de douze nouveaux États dont une majorité entendait retisser un substrat national, après des années d'occupation ou de domination. Toutefois, ce souci n'est pas incompatible avec la possibilité d'une avancée conjointe en matière artistique et culturelle. Si les États doivent renoncer au sacro-saint rayonnement culturel (qu'il soit ou non étroitement lié à des objectifs économiques), ils ont, dans le cadre de l'Union européenne, la possibilité de mettre en commun leurs attentes et leurs priorités. Ainsi, après la Seconde Guerre mondiale 
et quelques années d'un urbanisme destructeur, le thème du patrimoine commun européen est-il apparu comme une plate-forme de ralliement et a donné lieu à des programmes dès les années 80 . De même, la nécessité de conforter les échanges et coopérations universitaires a été affirmé par les États membres dès 1977. Enfin, le cinéma et l'audiovisuel ont bénéficié, dès 1984, d'une attention soutenue, conduisant à la mise en place de directives tout comme à la création du programme MEDIA. Ces premiers pas ont généré le développement de partenariats entre associations, sociétés de production indépendantes, collectivités territoriales, organisations paraétatiques et universités. À titre d'exemple, des centaines de bibliothèques publiques européennes travaillent avec la Commission européenne depuis 1970 (Barret-Durocq, 1992)'.

\section{Différencier État et nation}

Après la création du Conseil «Culture et audiovisuel », les États membres ont pris l'habitude d'envoyer leurs représentants dans des réunions de suivi des programmes (comités de gestion), mais aussi pour arrêter des décisions communes sur un certain nombre de questions. En 1994, l'obtention de l'unanimité des États membres pour l'exception culturelle n'est pas le moindre résultat de ces concertations, connaissant par exemple, la difficulté des Britanniques à s'y rallier (Jones, 1999). Autrement dit, le statut des États membres a été lui-même transformé par cette fréquentation communautaire, ce qu'Ulrich Beck et Edgar Grande (2004) appellent un «méta-pouvoir ». En 1992, la Charte du Conseil de l'Europe sur les langues régionales et minoritaires en Europe, puis le ralliement de l'Union européenne à la rédaction de la Convention UNESCO ont permis d'échanger de nombreuses réflexions comparatives sur le sort des cultures et langues minoritaires dans les États membres. Si l'ouvrage Differing diversities, coordonné parTony Bennett (200I), a mis en évidence le faible impact des politiques culturelles nationales entreprises à l'endroit de la diversité culturelle - et notamment en ce qui concerne les migrants -, diverses contributions ont mis à jour une nouvelle idée de l'État qui ne serait pas « nation » mais l'alliance de plusieurs nations. Les expériences britannique, espagnole, mais aussi belge et italienne ont été étudiées dans une optique de coexistence entre État et autonomies régionales. Cette brèche - plus grande que prévu en France - dans l'idée de l'État homogène a été relayée par la situation des États d'Europe centrale et orientale. Mosaïques sans précédent de minorités, théâtres

\footnotetext{
Les collaborations ont commencé sous l'égide de la Direction générale XIII de la Commission européenne (alors en charge des Télécommunications, des industries de l'information et de l'innovation).
} 
de déplacement de populations et de recomposition continuelle d'États depuis le début du $X X^{e}$ siècle, les nouveaux États Est-européens ont assumé officiellement leur composante multiculturelle et multilinguistique? Beaucoup d'États européens se sont donc découverts « autres 》, même si cette altérité assumée publiquement présente d'infinies restrictions et limites. Un autre exemple de la mobilisation dynamique des États est le RIPC (Réseau international pour la politique culturelle) : regroupant des États du monde entier aussi différents que la Côte d'Ivoire, la Corée du Sud et la Suisse, il a accompagné les travaux de la Convention uNESCO et fait des préconisations sur le futur instrument juridique international sur la diversité culturelle. Si les autorités canadiennes se sont montrées réticentes à l'idée de confier ce chantier à l'UNESCO, elles n'en ont pas moins joué leur partition avec beaucoup d'efficacité par rapport à la Convention?3. Quant à I'Union européenne, elle a été la première union régionale à adopter la Convention dont la Commission européenne a accompagné la préparation avec beaucoup de rigueur. Enfin, il n'est sans doute pas inutile de rappeler que la « diversité culturelle et linguistique » fait partie des objectifs du futur Traité sur le fonctionnement de l'Union européenne, ouvrant ainsi la possibilité d'actes de jurisprudence auprès de la Cour de Justice des Communautés européennes. II reste que le cadre juridico-institutionnel instauré dans le cadre de l'Union européenne a vocation à être contesté, voire subverti, par les acteurs culturels et artistiques. D'où l'importance de l'émergence des nombreux réseaux et associations qui maillent le territoire communautaire.

L'horizon communautaire a suscité depuis les années 80, la création de plusieurs centaines de réseaux d'associations paneuropéennes dont la vivacité et la capacité de proposition ont eu des incidences sur l'évolution des programmes MEDIA et CULTURE pour ne citer que ces exemples (Autissier, 2003). Enfin et peut-être surtout, l'intégration européenne a donné des ailes aux échelons supra-nationaux, notamment les régions et les communes. On ne compte plus aujourd'hui les réseaux et associations européennes et internationales créées par ces entités: Conseil des communes et régions d'Europe (CCRE), Eurorégions, Eurocités/Eurocities, Assemblée des régions d'Europe, Les rencontres, Conférence des Villes de l'Arc atlantique, Union des villes baltiques, Villes-Refuges, Cités et gouvernements locaux unis (CGLU), Villes de l'Agenda 2 I pour la Culture, etc.

2 Ainsi en est-il de la nomination de Sibiu, ville peuplée de Roumains d'origine saxonne, en tant que Capitale culturelle européenne représentant la Roumanie en 2007.

3 C'était notamment la position de Sheila Copps, ministre du Patrimoine du Canada au tournant des années 90. 


\section{Vers un agenda culturel renouvelé}

La Commission Barroso a pris en compte ces évolutions dans sa Communication relative à unAgenda culturel européen à l'ère de la mondialisation ${ }^{4}$. Cette communication propose une stratégie paradoxale, à la fois fondée sur la coopération accrue entre États membres et un travail continu avec la «société civile culturelle européenne ». Reconnaissant la dimension transversale de la culture (au sens anthropologique et artistique) dans le développement territorial et l'entrée dans une « société de l'information et de la connaissance », ce document propose aux États membres de recourir à la Méthode ouverte de coordination (MOC) qui a déjà fait ses preuves en matière de jeunesse, d'éducation et de formation professionnelle. Ainsi, écrivent ses rédacteurs, les États se sentiront-ils valorisés et plus enclins à engager à quelques-uns des initiatives d'envergure. Faut-il rappeler que l'efficacité du passage au LMD (Processus de Bologne) résulte d'une MOC aujourd'hui suivie par 37 États européens ${ }^{5}$ ? Loin de « boucler la boucle », la Commission européenne fait cette proposition à des États dont certains ont acquis une expérience multilatérale communautaire. La mobilisation des ministres de la Culture allemand, français, hongrois et espagnol sur ces questions à travers des conférences annuelles, a d'ailleurs démontré que le moment était propice pour une relance de la coopération culturelle européenne $^{6}$. La Commission n'oublie pas d'associer potentiellement les collectivités territoriales à cette coopération à géométrie variable7. Symétriquement, elle revendique un travail régulier avec les représentants de la société civile culturelle, à charge pour cette dernière de se rendre « représentative », ce qui n'est pas le moindre problème. En effet, comme le soulignent les rédacteurs de la communication, les troupes artistiques et culturelles européennes restent extrêmement fragmentées et - tout comme les politiques culturelles nationales - regroupées autour d'objectifs sectoriels même si une nouvelle génération de plateformes numériques ou la création d'alliances nationales pour la diversité culturelle ont facilité des convergences. Ainsi les bataillons européens du Conseil mondial des cultures que Jean Tardif appelle de ses vœux, ne sont-ils peut-être pas si absents, comme je me suis efforcée de le démontrer.

${ }^{4}$ Communication de la Commission au Parlement européen, au Conseil, au Comité économique et social européen et au Comité des Régions. COM/2007/242 final// 0/05/2007. Adoptée le 16 nov. 2007 par le Conseil et le Parlement européen.

5 Même si l'on peut s'interroger sur le contenu de cette harmonisation en termes de savoirs académiques fondamentaux (voir Stamelos, 2003).

6 « Une âme pour l'Europe », Berlin, 2004 ; « Les Rencontres pour l'Europe de la Culture », Paris, 2005...

7 Même si l'association de grandes villes, Eurocités/Eurocities, regrette que la communication reste trop timide en la matière, laissant aux États le soin d'associer "leurs" entités territoriales. 
Enfin, la Communication de la Commission européenne recommande une articulation systématique entre coopération culturelle intra-européenne et coopération culturelle internationale ${ }^{8}$. Cette nouveauté est d'importance car elle permettra à la Direction générale Éducation et Culture de jouer un rôle transversal en matière de coopération culturelle extérieure, ce qui n'était pas toujours le cas dans l'ancienne gestion des programmes opérée par EuropeAid sous la supervision de quatre commissaires mais pas celui de la culture. La fluidité escomptée des relations entre Société de l'information et médias, d'une part, et Culture et éducation, d'autre part, avec EuropeAid, devrait favoriser une technicité plus grande en matière de programmes et des complémentarités efficaces?

\section{Pour une Europe cosmopolitique}

Faut-il craindre une dilution des activités culturelles européennes dans une mondialisation envahissante ? L'expérience montre que c'est l'expérience des guerres européennes et mondiales qui a forgé le sentiment de solidarité communautaire. De même, la mobilisation européenne en faveur des industries de programme du continent a été en quelque sorte aiguillonnée par la concurrence américaine. La décolonisation a conduit la Communauté européenne à mettre en place des accords ACP (Afrique-Caraïbes-Pacifique) ouverts à la culture. Ainsi I'Union européenne est-elle aujourd'hui l'un des premiers financeurs du cinéma et de l'audiovisuel d'Afrique sub-saharienne. Enfin, comme Denis de Rougemont (1962) l'avait déjà remarqué, le partage européen s'exprime excellemment par ou à travers une confrontation avec les autres aires géoculturelles. D'ores et déjà, les programmes MEDIA 2007 et CULTURE sont ouverts à des ressortissants de pays tiers. La Commission européenne a mis en place un appel à propositions pour des projets Union européenne/Chine et Inde en 2007 et s'apprête à réitérer l'exercice pour la coopération artistique et culturelle de ressortissants de l'Union avec des professionnels d'Amérique latine. Notons également l'intérêt pour la coopération culturelle et audiovisuelle euro-asiatique de la création de la Fondation Europe-Asie à Singapour, alimentée par un fonctionnement intergouvernemental dans lequel la Commission est l'un des partenaires à côté des États membres, des États membres de l'ANASE/ASEAN, de la Chine, du Japon et de I'Inde. Signalons également, sur un modèle comparable,

8 Cette recommandation s'applique tout au moins aux ressortissants des pays tiers ayant signé avec la Commission européenne, des accords incluant la culture.

9 À l'instar de ce qui existe pour la programme Jeunesse en action qui, au titre de « L'Éducation et la Culture », gère le programme de coopération euro-méditerranéenne dans le domaine de la jeunesse par ailleurs mis en place dans le cadre du Processus de Barcelone. 
la naissance de la Fondation Anna Lindh à Alexandrie qui a pour mission d'opérer un rapprochement des sociétés civiles de I'Union et de la Rive Sud de la Méditerranée. N'oublions pas non plus le programme ErasmusMundus qui permet la création de consortia entre universités de l'Union et universités de tous les continents. Bien entendu, ces initiatives ne sont pas toutes frappées du sceau de l'efficacité et de l'accessibilité. Comme le fait remarquer Jean Tardif à propos du futur traité sur le fonctionnement de l'Union européenne, on ne peut s'empêcher de critiquer la complexité des procédures administratives, le caractère extrêmement contraignant des rapports à remettre à la Commission européenne... Et parfois aussi, le manque de réalisme de certains programmes communautaires plaqués sur une réalité qui les rend inopérants. Pourtant, la dynamique instaurée par la Commission européenne devrait nous permettre d'augurer de développements positifs.

Parallèlement, l'injonction de la diversité culturelle est de plus en plus prise au sérieux par des pays importants. Ainsi, le Brésil, certes rallié tardivement à la cause de la diversité culturelle, en a-t-il fait un objet de politique publique puisqu'il a créé en son ministère de la Culture un département de la Diversité culturelle, confié à une personnalité fort respectée, Sergio Mamberti, grand acteur brésilien. De même, le réalisateur Orlando Senna a pris la tête de la nouvelle chaîne publique de télévision brésilienne, projet visant à regrouper des activités existantes et à en créer de nouvelles pour que le Brésil ait enfin une chaîne publique digne de ce nom, et reflétant le pluralisme culturel de ce pays. Ajoutons que les concepteurs du projet ont engagé toute une série de prospections internationales - notamment en Amérique latine - pour que la nouvelle station soit aussi le reflet des productions de tout le sous-continent. De même, des producteurs indiens de cinéma développent actuellement des coproductions avec des professionnels européens pour valoriser de nouveaux croisements imaginaires et mettre en commun des savoir-faire spécifiques (Kandar, 2007).

Pour en revenir aux aires géoculturelles telles que Jean Tardif les définit, ne serait-il pas souhaitable de capitaliser au profit des nouvelles configurations qu'il appelle de ses vœux, l'expérience d'un certain nombre d'unions régionales? On en compte une vingtaine aujourd'hui dans le monde (Ténier, 2003). Leur degré d'intégration reste en deçà de celui de la construction communautaire. Pourtant, certaines d'entre elles ont esquissé des stratégies culturelles ou éducatives qui méritent d'être signalées. Ainsi du Mercosur/Mercosul qui a renforcé l'apprentissage de l'espagnol au Brésil dans l'enseignement secondaire, tout comme celui du portugais dans les États partenaires hispanophones. Citons également l'accord de coproduction signé en 2007 entre le Brésil et l'Allemagne sous l'égide de la Commission Cinéma et Audiovisuel du Mercosur, 
et prévoyant qu'à terme - et si les États concernés le ratifient - tout avantage conféré par cet accord aux ressortissants brésiliens peut l'être aux professionnels membres du Mercosur. L'objectif étant le même pour les professionnels allemands et ressortissants de I'Union européenne. La sICA (Sistema de la Integración Centroamericana/Système d'intégration centre-américaine) a créé une université centre-américaine et mis en place et des équivalences de diplômes. Ces initiatives multilatérales sont relayées par le dynamisme des professionnels : association centreaméricaine de professionnels de l'audiovisuel et du cinéma, association centre-américaine de professionnels du patrimoine... Citons encore la CARICOM (Carribean Community and Common Market/Communauté et Marché commun des Caraïbes), laquelle, malgré la pauvreté de ses États membres, s'efforce de promouvoir ses techniques de médecine traditionnelle et ses industries musicales, avec l'objectif de créer des pôles de production à l'échelle régionale. Quant à la SADC (Southern African Development Community/Communauté de développement de I'Afrique australe), elle a mené une réflexion sur les politiques culturelles - en particulier les industries culturelles - à l'échelle de tous ses États membres. Enfin, la SAARC (South Asian Association for Regional Cooperation/Association de coopération régionale d'Asie du Sud) a lancé, en 2004, un Plan d'action pour les télécommunications et technologies de l'information et de la communication, à l'échelle régionale.

\section{Placer l'amateur au cœur d'une nouvelle société cognitive}

La Commission européenne a créé une direction générale Société de l'information et médias, regroupant un certain nombre de compétences préexistantes, ainsi que la tutelle des programmes audiovisuels et numériques, en relation avec le Programme-cadre de recherche et développement technologique (PCRDT). Sa page d'accueil ne laisse guère de doutes sur l'importance du sujet, au regard des instances communautaires: "Informatique, télécommunications et audiovisuel représentent 8 \% du PIB actuel de I'Union européenne, soit deux fois plus qu'au début des années quatre-vingt-dix et emploient treize millions de personnes ». Selon la direction générale, les enjeux de la société de l'information et des médias sont pris en charge à travers trois types d'actions : la réglementation, la recherche et que les programmes. Malgré la priorité officiellement donnée à ce secteur, relayant les objectifs de la stratégie de Lisbonne (2000), I'on est régulièrement surpris par la façon dont la Commission européenne réduit la réflexion sur les nouvelles technologies à une affaire de rentabilité immédiate. Sur ce point, l'exemple donné par Joëlle Farchy est très caractéristique : la recommandation du 
I 8 octobre 2005, relative à la délivrance d'une licence pan-européenne pour la distribution de musique en ligne a en effet provoqué un tollé parmi les organisations européennes et internationales concernées : CISAC, GESAC, Aepo-Artis, Fédération internationale des musiciens... La logique strictement comptable ne tient pas, face aux responsabilités multiples et notamment sociales dont sont investies les sociétés de gestion collective ${ }^{10}$. En même temps, Joëlle Farchy a raison de dénoncer l'instrumentalisation des droits d'auteur et droits voisins en une rente de situation pour les majors. Le prolongement de la durée des droits voisins à soixante-dix ans, sur lequel travaille actuellement la Commission européenne provoque des réactions contradictoires au sein des milieux professionnels : d'une part, elle rend justice à une revendication de longue date des professionnels et de leurs représentants. D'autre part, étant donné la crise actuelle des revenus du milieu musical, les sommes à redistribuer seront - sauf exception - relativement faibles. On voit là l'un des effets contradictoires du développement technologique qui rend très vite obsolètes les dispositions régulatrices antérieures. De même, la crispation des majors et des producteurs sur les DRM (Digital Rights Management) s'est révélée peu efficace face aux hackers de tout acabit. Certaines plate-formes numériques commerciales ne fondentelles pas aujourd'hui leur promotion sur l'absence de DRM (Culture Europe International, 2007) ? Quant à la volonté de la Commission européenne de revenir sur la rémunération pour copie privée, au motif de ne pas taxer deux fois les consommateurs, elle a dû être provisoirement enterrée en décembre 2006, par Manuel Barroso lui-même. Mais un nouveau front s'est ouvert lors de la modification de l'ex-directive «Télévision sans frontières ». L'ensemble des supports concernés (numériques ou non) est désormais désigné par le vocable de « services de médias audiovisuels », à charge pour eux de «favoriser, chaque fois que cela est réalisable, la distribution et la production d'œuvres européennes et [de] promouvoir ainsi activement la diversité culturelle ». La principale conséquence de ces nouvelles obligations pour les pourvoyeurs d'accès et de contenus, est le renoncement, dans la directive, à une réglementation détaillée « en matière d'insertion de spots publicitaires », au motif de ne pas pénaliser exagérément un marché en plein essor. Inutile de préciser que ces nouvelles dispositions ont fait couler beaucoup d'encre.

Le plus grave est sans doute que, comme le note Joëlle Farchy, ni les pouvoirs nationaux, ni l'Union européenne, ni même les organisations multilatérales n'ont pris la mesure de la révolution anthropologique générée par les usages généralisés de l'internet et de la compression

\footnotetext{
${ }^{10}$ A.-M. Autissier, A. du Port de Pontcharra, L'action sociale et culturelle des SPRD en Europe: un modèle pour les stratégies culturelles?, site de l'ADAMI : www.adami.fr
} 
numérique. «La possibilité de court-circuiter une culture survalorisée par le marché, ou par des institutions publiques aux experts déconnectés, est en soi une chance pour la diversité culturelle », écrit Marc Le Glatin (2007 : 80). Cet auteur parie sur la « confiance entre des internautes en interaction permanente ». II imagine également que le développement multiforme de l'internet appellera, en contrepartie, une nouvelle demande de spectacles vivants et de rencontres de tout type. Ces réflexions sur la capacité des internautes à renouer un contact direct avec les œuvres et celle des artistes et des médiateurs indépendants à en profiter, appelle quelques remarques: même si elle est incomparable avec celle qui prévalait voilà un siècle, la fréquentation des institutions culturelles alternatives ou non - marque le pas dans l'ensemble des pays européens. Dans le domaine cinématographique l'Union européenne produit, à elle seule, plus de films de long métrage que les États-Unis. Pourtant, comme le souligne Joëlle Farchy, les montants consentis à la promotion restent faibles et les marchés nationaux fragmentés, tout en étant dominés par la production des majors américaines. Si les efforts de MEDIA ont fait passer le pourcentage de circulation des films et programmes audiovisuels européens de quelque $6 \%$ en 1989 à $22 \%$ en 2006, la fréquentation cinématographique plébiscite en majorité des produits de «l'hyperculture globalisante », selon l'expression de Jean Tardif - qu'ils soient américains, français ou de Hong-Kong. La fréquentation d'un cinéma national indien par le public indien tient d'abord au protectionnisme soigneusement orchestré par les autorités et les distributeurs indiens. Mais les films bollywoodiens relèvent en majeure partie d'un formatage qui n'illustre en rien la diversité culturelle et la réalisatrice de Water'", Deepa Mehta, a dû tourner son film au Sri Lanka. Ainsi souscrivonsnous parfaitement aux conditions de la diversité culturelle, telle que Joëlle Farchy les énonce. L'une des grandes questions posées par cette analyse est le vouloir du public lui-même. Certes, il existe différents publics pour différents types d'œuvres et internet peut favoriser cette variété. Pourtant les plateformes musicales se caractérisent par une homogénéité de l'offre ${ }^{12}$. L'un des arguments les plus discutés depuis une décennie et qui légitime les politiques culturelles publiques est celui de l'accès : proximité, baisse des prix, fidélisation par abonnement, offre pluridisciplinaire dans un même lieu, investissement d'espaces a priori non culturels, festivalisation... Tout aura été fait, ou presque, pour rapprocher les œuvres des publics, pense-t-on. Parallèlement, on se sera trop peu soucié des motivations des publics. L'enquête du sociologue

\footnotetext{
II Le film Water est sorti en 2005. II relate l'enfermement à vie d'une très jeune veuve dans l'Inde de 1938.

12 Étude et synthèse du rapport fING : Musique et numérique, la carte de l'innovation, 2007. Site de l'ADAMI : wWw.adami.fr
} 
Bernard Lahire (2004) décrit la multiplicité des paramètres collectifs et individuels qui facilitent ou freinent la relation avec la culture. Les modes de détournement, d'appropriation évoqués par Joëlle Farchy ont été trop peu sondés, et surtout de façon insuffisamment qualitative. En revanche, il est vrai que la télévision et la radio ont fait plus pour la démocratisation culturelle que n'importe quelle forme de politique publique. Aujourd'hui, l'internet prend le relais, mettant en péril les revenus des majors mais facilitant également un contrôle central des données, comme le remarque Joëlle Farchy. Les communautés universitaires et artistiques se sont rapidement approprié un outil que les scientifiques avaient d'ailleurs contribué à créer et une certaine utopie informationnelle - de partage et de mutualisation du savoir et de la création a fait son chemin (Flichy, 200I), comme en témoigne l'expérience des Licences libres. II reste que, sans accompagnement éducatif, on ne voit guère comment la toile contribuera per se à générer une disparité des attentes et des contenus. Selon Bernard Stiegler (2005 : 124), l'Europe doit inventer de nouveaux modèles de socialisation des technologies culturelles et cognitives, ce qu'il appelle « les technologies de l'esprit »: « La constitution de technologies industrielles de l'esprit suppose de dépasser la figure du consommateur, et de retrouver une intelligence de la figure de l'amateur qu'il s'agit de mettre au cœur du modèle industriel. L'absence de théorisation de l'immense potentiel social des nouvelles technologies est encouragée «par les groupes qui jouissent de rentes de situation [...] en particulier les industries de programme ».

\section{Conclusion}

Pour paraphraser Michel Wieviorka (200I), on pourrait en lieu et place de l'actuelle diversité culturelle, promouvoir une diversité culturelle 《 intégrée $»^{13}$. Fondée sur une alliance entre culture, éducation, recherche et politique économique, cette diversité culturelle ou plutôt ce pluralisme culturel envisagerait le développement des activités culturelles et artistiques à l'aune des droits culturels en tant qu'élément indivisible des droits de l'homme. Par conséquent, la révolution technologique en cours rendrait possible un accès individualisé et multi-supports à différents contenus culturels, informatifs ou artistiques. Elle permettrait également d'enrichir les différents messages ou supports sur un mode mutualisé. À l'échelle du monde, la mise en réseau d'initiatives locales - déjà engagée - permettrait la création de cet « écosystème symbolique » dont Jean Tardif souhaite l'avènement. De tels objectifs seront réalisables

\footnotetext{
${ }^{13}$ M. Wieviorka (200I) évoque le « multiculturalisme intégré » face au « multiculturalisme éclaté ».
} 
si l'on est capable de mettre en place de nouvelles formes de rétribution de la valeur intellectuelle et artistique. Mais ils ne se concrétiseront pas sans une réorientation des investissements publics et sans la mise en place de modèles économiques alternatifs aux oligopoles des industries culturelles.

\section{Références}

Autissier A.-M., 2002, «Les États, protagonistes ambigus de la coopération culturelle européenne », pp. 365-383, in : Roche Fr., dir., La culture dans les relations internationales, École française de Rome.

- 2003, «Cultural networks in Europe », pp. 21-27, in : Artists sharing without frontiers, Networking in the Enlargment Process, European Council of Artists Report, Copenhague, Eureopan Council of Artists.

Barret-Ducrocq Fr., dir., 1992, Traduire l'Europe, Paris, Payot.

Beck U., Grande E., 2004, Pour un empire européen, trad. de l'allemand par A. Duthoo, Paris, Flammarion, 2007.

Bennett T., 200I, Differing diversities, Cultural policy and cultural diversity, Strasbourg, Éd. du Conseil de l'Europe.

Culture Europe International, 2007, «L'épreuve de réalité des DRM», dossier « Réinventer l'exception culturelle à l'ère numérique », Hors-série, print.-été.

Flichy P., 200 I, L'imaginaire d'internet, Paris, Éd. La Découverte.

Jones B. E., 1999, « Le cas britannique », Culture Europe, 25, févr., pp. 6-8, www. culture-europe-international.org

Kandar M., 2007, Planète India. L'ascension turbulente d'un géant démocratique, trad. de l'américain par A. R. Lewin, Arles, Éd. Actes Sud, 2008.

Lahire B., 2004, La culture des individus, Dissonances culturelles et distinction de soi, Paris, Éd. La Découverte.

Le Glatin M., 2007, Internet : un séisme dans la culture?, Mercues, Éd. de l'Attribut.

Roche Fr., 2002, La culture dans les relations internationales, Rome, École française de Rome.

Rougemont de D., 1962, Le dialogue des cultures, Neuchâtel, Éd. de la Baconnière.

Stamelos Y., 2003, «La construction d'un espace européen d'enseignement supérieur : rétrospective, situation actuelles et perspectives », Revue des sciences de l'éducation, Vol. XXIX, 2, pp. 277-296.

Stiegler B., 2005, Constituer l'Europe, 2. Le motif européen, Paris, Galilée.

Ténier J.-J., 2003, Intégrations régionales et mondialisation. Complémentarité ou contradiction, Paris, Éd. La Documentation française.

Wieviorka M., 200I, La différence, Paris, Balland. 